\title{
Thermal properties and physicochemical behavior in aqueous solution of pyrene-labeled poly(ethylene glycol)-polylactide conjugate
}

This article was published in the following Dove Press journal:

International Journal of Nanomedicine

8 April 2015

Number of times this article has been viewed

\author{
Wei-Lin Chen ${ }^{1,2}$ \\ Yun-Fen Peng ${ }^{1,3}$ \\ Sheng-Kuo Chiang' \\ Ming-Hsi Huang ${ }^{1-3}$ \\ 'National Institute of Infectious \\ Diseases and Vaccinology, National \\ Health Research Institutes, Miaoli, \\ Taiwan; ${ }^{2}$ Graduate Institute of Life \\ Sciences, National Defense Medical \\ Center, Taipei, Taiwan; ${ }^{3} \mathrm{PhD}$ Program in \\ Tissue Engineering and Regenerative \\ Medicine, National Chung Hsing \\ University, Taichung, Taiwan
}

\begin{abstract}
A fluorescence-labeled bioresorbable polymer was prepared by a coupling reaction of poly(ethylene glycol)-polylactide (PEG-PLA) with carboxyl pyrene, using N,N'diisopropylcarbodiimide/1-hydroxy-7-azabenzotriazole (DIC/HOAt) as a coupling agent and 4-dimethylaminopyridine (DMAP) as a catalyst. The obtained copolymer, termed PEG-PLApyrene, was characterized using various analytical techniques, such as gel permeation chromatography (GPC), matrix-assisted laser desorption/ionization time-of-flight mass spectrometry (MALDI-TOF MS), proton nuclear magnetic resonance ( $\left.{ }^{1} \mathrm{H}-\mathrm{NMR}\right)$, infrared spectroscopy (IR), differential scanning calorimetry (DSC), and thermogravimetric analysis (TGA), to identify the molecular structure and to monitor the thermal property changes before and after the reaction. The presence of a pyrene moiety at the end of polylactide (PLA) did not alter the crystallization ability of the poly(ethylene glycol) (PEG) blocks, indicating that the conjugate preserved the inherent thermal properties of PEG-PLA. However, the presence of PEG-PLA blocks strongly reduced the melting of pyrene, indicating that the thermal characteristics were sensitive to PEG-PLA incorporation. Regarding the physicochemical behavior in aqueous solution, a higher concentration of PEG-PLA-pyrene resulted in a higher ultraviolet-visible (UV-vis) absorbance and fluorescence emission intensity. This is of great interest for the use of this conjugate as a fluorescence probe to study the in vivo distribution as well as the internalization and intracellular localization of polymeric micelles.
\end{abstract}

Keywords: bioresorbable polymer, fluorescence probe, pyrene, polymeric micelles, poly (ethylene glycol)-polylactide, PEG-PLA

\section{Introduction}

Amphiphilic block copolymers of poly(ethylene glycol) (PEG) and polylactide (PLA) have attracted substantial attention for the sustained delivery of biologically active agents due to their biocompatibility and bioresorbability. ${ }^{1-7}$ Studies have been performed to investigate the degradability and permeability of PEG/PLA-based vehicles, such as hydrogels, ${ }^{1,2}$ micelles, ${ }^{3,4}$ micro/nanoparticles,${ }^{5}$ and emulsions, ${ }^{6,7}$ among others. Ideally, a bioresorbable delivery vehicle must degrade and resorb in vivo at a predefined rate, allowing the bioactive agents to either attach at the surface or be entrapped within the microenvironment before administration and be sequentially released postadministration..$^{1-3,5-7}$ Attempts have been made to investigate the in vivo distribution, to elucidate the targeting delivery of bioactive candidates bearing fluorescence. ${ }^{5,8}$ Moreover, understanding the internalization and intracellular localization of the bioactive candidates after formulation with these types of vehicles is useful to elucidate the biological interactions and the mechanism of enhanced therapeutic efficacy of the targeted delivery.
Correspondence: Ming-Hsi Huang National Institute of Infectious Diseases and Vaccinology, National Health Research Institutes, Number 35 Keyan Road, Zhunan Town, Miaoli County 35053, Taiwan

Tel +886 37246 I66 ext 37742

Fax +886 37583009

Email huangminghsi@nhri.org.tw 
Pyrene and its derivatives have been widely used as fluorescence probes and as models of drugs with poor water solubility. ${ }^{8-11}$ Due to pyrene's polycyclic aromatic structure, it is highly stable, with a high quantum yield and lifetime. ${ }^{11}$ In this study, we report the synthesis of a PEG-PLA copolymer bearing a fluorescence marker, obtained by ring-opening polymerization of DL-lactide in the presence of monomethoxy PEG followed by the coupling reaction with carboxyl pyrene. The obtained PEG-PLA-pyrene copolymer was characterized by gel permeation chromatography (GPC), matrix-assisted laser desorption/ionization time-of-flight mass spectrometry (MALDI-TOF MS), proton nuclear magnetic resonance ( $\left.{ }^{1} \mathrm{H}-\mathrm{NMR}\right)$, infrared spectroscopy (IR), differential scanning calorimetry (DSC), and thermogravimetric analysis (TGA). The physicochemical behavior of PEG-PLA-pyrene in aqueous solution was investigated by ultraviolet (UV)-visible (vis) absorption spectroscopy, fluorescence spectroscopy, and dynamic light scattering (DLS) technology. The results were compared with those obtained from different polymer precursors as described in the literature. ${ }^{4,8}$

\section{Materials and methods}

\section{Materials}

DL-lactide was purchased from Sigma-Aldrich Corp (St Louis, MO, USA) and was recrystallized from ethyl acetate. Polyethylene glycol 2000 monomethyl ether $\left(\mathrm{MePEG}_{2000}\right.$ ) was supplied by Fluka (Buchs, Switzerland) and was used as received. Tin(II) 2-ethylhexanoate $\left(\mathrm{SnOct}_{2}\right)$, deuterated chloroform, $\alpha$-cyano-4-hydroxycinnamic acid (CHCA), and sodium trifluoroacetate (Na-TFA) were purchased from Sigma-Aldrich Corp. 1-Pyrenebutyric acid was purchased from Alfa Aesar (Ward Hill, MA, USA). N,N'-diisopropylcarbodiimide (DIC), 1-hydroxy-7-azabenzotriazole (HOAt), and 4-dimethylaminopyridine (DMAP) were purchased from Merck KGaA (Darmstadt, Germany). All of the solvents were of analytical grade.

\section{Synthesis}

The AB-type diblock copolymer PEG-PLA was synthesized by ring-opening polymerization of DL-lactide in the presence of $\mathrm{MePEG}_{2000}$ and $\mathrm{SnOct}_{2}$, as previously described. ${ }^{7}$ In a typical synthesis, DL-lactide $(4 \mathrm{~g}), \mathrm{MePEG}_{2000}(8 \mathrm{~g})$, and $\mathrm{SnOct}_{2}(30 \mathrm{mg})$ were introduced into a round-bottomed flask. Polymerization was performed under vacuum at $140^{\circ} \mathrm{C}$ for 24 hours. The obtained polymer was recovered by the dissolution/precipitation method, with acetone as the solvent and ethanol as the nonsolvent, followed by filtration and vacuum drying. The coupling reaction of PEG-PLA and 1-pyrenebutyric acid was performed with HOAt/DIC/DMAP in N-methyl-2-pyrrolidone at room temperature for 10 hours. The resulting PEG-PLA-pyrene was recovered by the precipitation method, with diethyl ether as the nonsolvent.

\section{Characterization}

GPC was performed by using a setting composed of an isocratic pump, a refractive index detector, and two sizeexclusion columns connected in series, one PLgel ${ }^{\mathrm{TM}} 5 \mu \mathrm{m}$ guard column $(7.5 \times 50 \mathrm{~mm})$, and one PLgel $5 \mu \mathrm{m}$ mixed-D column $(7.5 \times 300 \mathrm{~mm})$. The mobile phase was tetrahydrofuran, and the flow rate was $1.0 \mathrm{~mL} \cdot \mathrm{min}^{-1}$. The data were expressed relative to polystyrene standards (Varian Inc., Amherst, MA, USA). Mass spectra were acquired using a Micromass ${ }^{\circledR}$ MALDI micro MX ${ }^{\mathrm{TM}}$ Time of Flight Mass Spectrometer (Waters ${ }^{\circledR}$, Milford, MA, USA) in the reflection mode. An aliquot $(1 \mu \mathrm{L})$ of polymer samples was premixed with $1 \mu \mathrm{L}$ of $0.2 \%$ trifluoroacetic acid/acetonitrile and then mixed with CHCA as the matrix and Na-TFA as the dopant. An aliquot $(1 \mu \mathrm{L})$ of sample solutions was spotted on a MALDI sample plate and air-dried to form a thin matrix/ analyte film. ${ }^{1} \mathrm{H}-\mathrm{NMR}$ spectra were recorded at room temperature with a Varian VXR $300 \mathrm{MHz}$ spectrometer using deuterated chloroform as the solvent and tetramethylsilane as the shift reference. IR spectra were recorded with a Perkin Elmer Spectrum 100 FTIR Spectrometer (PerkinElmer, Santa Clara, CA, USA). DSC and TGA measurements were obtained on a SDT Q600 thermal analyzer (TA Instruments, New Castle, DE, USA) under nitrogen at a heating rate of $10^{\circ} \mathrm{C} \cdot \mathrm{min}^{-1}$. The polymeric micellar solutions in distilled water were incubated in 96-well quartz microplates. The absorbance and fluorescence measurements of the samples were obtained on a SpectraMax ${ }^{\circledR}$ M2 multimode plate reader (Molecular Devices LLC., Sunnyvale, CA, USA). The absorbance spectra were recorded from 300 to $600 \mathrm{~nm}$ with a width of $10 \mathrm{~nm}$. The fluorescence was measured using an excitation wavelength of $340 \mathrm{~nm}$, and the excitation and emission slits were $10 \mathrm{~nm}$ wide. The particle size of the polymer in aqueous solution was monitored by DLS technique (Brookhaven 90Plus Particle Size Analyzer, Brookhaven Instruments Corp, Holtsville, NY, USA).

\section{Results and discussion}

The schematic diagrams of the synthesis and the chemical structure of the block copolymers are shown in Figure 1. The PEG-PLA diblock copolymer was synthesized by ringopening polymerization of DL-lactide on monomethoxy PEG, resulting in an AB-type copolymer composed of a 

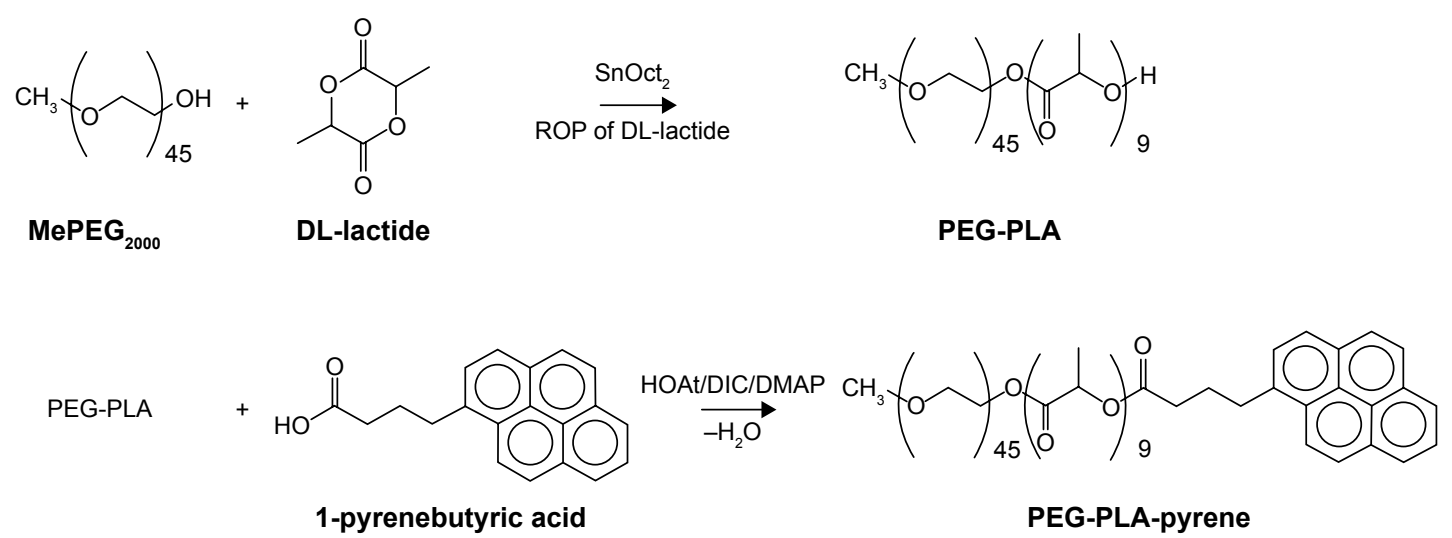

Figure I Synthesis scheme of PEG-PLA-pyrene.

Abbreviations: DIC, N,N'-diisopropylcarbodiimide; DMAP, 4-dimethylaminopyridine; HOAt, I-hydroxy-7-azabenzotriazole; MePEG 2000 , polyethylene glycol 2000 monomethyl ether; PEG-PLA, poly(ethylene glycol)-polylactide; ROP, ring-opening polymerization.

hydrophilic block of PEG and a lipophilic block of PLA, with a methoxy group at the PEG terminal and a hydroxyl group at the PLA end. PEG-PLA-pyrene was synthesized by coupling PEG-PLA with a carboxyl pyrene and 1-pyrenebutyric acid, using DIC/HOAt as a coupling agent and DMAP as the catalyst. Table 1 summarizes the molecular characteristics of 1-pyrenebutyric acid, PEG-PLA, and PEG-PLA-pyrene.

Figure 2A presents the GPC traces of PEG-PLA-pyrene, PEG-PLA, and the corresponding fluorescence labeling reagent carboxyl pyrene. PEG-PLA-pyrene exhibited a single peak with a narrow molecular weight distribution, indicating the absence of unreacted 1-pyrenebutyric acid. MALDI-TOF MS was used to measure the detailed molecular weight of the pyrene-labeled PEG-PLA block copolymer. As shown in Figure 2B, the PEG-PLA spectra were well-resolved, and the peaks were separated by 44 and 72 mass units, which corresponded to the molecular weight of the OE motif (oxyethylene units $=44.03 \mathrm{~g} \cdot \mathrm{mol}^{-1}$ ) and LA motif (lactyl units $=72.06 \mathrm{~g} \cdot \mathrm{mol}^{-1}$ ), respectively. The molecular weight of PEG-PLA ranged from 1,500 to 2,800 $\mathrm{g} \cdot \mathrm{mol}^{-1}$. After coupling with 1-pyrenebutyric acid, the molecular weight distribution of the resulting polymer shifted to $1,800-3,200 \mathrm{~g} \cdot \mathrm{mol}^{-1}$, which indicated the chain extension of the pyrene moiety onto PEG-PLA. Figure 2C shows the ${ }^{1} \mathrm{H}-\mathrm{NMR}$ spectrum of the PEG-PLA-pyrene block copolymer in deuterated chloroform. Typical signals of the PLA and PEG components were observed, as follows: signals at $1.5\left(-\mathrm{CH}_{3}\right)$ and $5.2 \mathrm{ppm}$ $(-\mathrm{CH})$ were assigned to PLA blocks and the signal at 3.6 ppm $\left(-\mathrm{CH}_{2}-\right)$ to PEG blocks. ${ }^{2}$ The coupling of pyrene was confirmed by the appearance of an aromatic proton peak area between 7.8 and $8.6 \mathrm{ppm}^{4}$, and the absence of a peak at $12.2 \mathrm{ppm}(-\mathrm{OH})$ for the starting carboxyl pyrene. The PEG/ PLA weight ratio of PEG-PLA-pyrene ( $25 \mathrm{wt} \%$ PLA content) was determined from the integrations of the signals due to PEG blocks at $3.6 \mathrm{ppm}$ and to PLA blocks at $1.5 \mathrm{ppm}$. The same method was used to calculate the pyrene/PEG-PLA ratio (the molar ratio of pyrene conjugated to the PEG-PLA chain) was about $70 \%$, from the ratio of the area of PLA resonances at $1.5 \mathrm{ppm}$ to the area of pyrene resonances between 7.8 and $8.6 \mathrm{ppm}$. Figure 2D shows the IR spectra of 1-pyrenebutyric acid, PEG-PLA, and the corresponding PEG-PLA-pyrene. 1-Pyrenebutyric acid exhibited bands at 3,052 and 1,694 $\mathrm{cm}^{-1}$ due to $\mathrm{OH}$ and $\mathrm{C}=\mathrm{O}$ stretching of carboxylic acid groups,

Table I Molecular characteristics of I-pyrenebutyric acid, PEG-PLA, and PEG-PLA-pyrene

\begin{tabular}{|c|c|c|c|c|c|c|c|c|}
\hline Name & $\mathbf{W}_{\mathrm{PEG}} / \mathbf{W}_{\mathrm{PLA}}^{\mathrm{a}}$ & $M_{n}^{b}(g / m o l)$ & $M_{w} / M_{n}^{b}$ & $M_{n}^{c}(g / m o l)$ & $\Delta H_{m}{ }^{d}(J / g)$ & $\mathbf{T}_{m}{ }^{d}\left({ }^{\circ} \mathrm{C}\right)$ & $\mathrm{T}_{\mathrm{d}}{ }^{\mathrm{e}}\left({ }^{\circ} \mathrm{C}\right)$ & $\begin{array}{l}\text { Particle } \\
\operatorname{size}^{f}(n m)\end{array}$ \\
\hline I-Pyrenebutyric acid & $\mathrm{N} / \mathrm{A}$ & 190 & 1.02 & 288 & 100 & 187 & 290 & nd \\
\hline PEG-PLA & 3.0 & 3,900 & 1.09 & $1,500-2,800$ & 100 & 46 & 240 & $9 \pm 3$ \\
\hline PEG-PLA-pyrene & 3.1 & 4,300 & 1.09 & $1,800-3,200$ & $100,9 g$ & $44,172^{g}$ & 190 & $I I \pm I$ \\
\hline
\end{tabular}

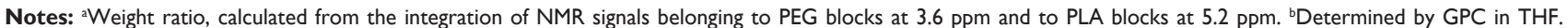
'Evaluated by MALDI-TOF. 'Measured by DSC. eDetected by TGA. fMonitored by DLS (mean \pm STD). ${ }^{8}$ Double melting peaks.

Abbreviations: $\Delta \mathrm{H}_{m}$, melting enthalpy; DLS, dynamic light scattering; DSC, differential scanning calorimetry; GPC, gel permeation chromatography; $\mathrm{M}_{\mathrm{n}}$, number average molecular weight; $M_{w}$, weight average molecular weight; MALDI-TOF, matrix-assisted laser desorption/ionization time-of-flight mass spectrometry; N/A, not applicable; nd, non detectable; NMR, nuclear magnetic resonance; PEG, poly(ethylene glycol); PLA, polylactide; STD, standard deviation; $T_{d}$, decomposition temperature; $T_{m}$, melting temperature; TGA, thermogravimetric analysis; THF, tetrahydrofuran; $\mathrm{W}_{\mathrm{PEG}}, \mathrm{W}_{\mathrm{PLA}}$, weight ratio of PEG block to PLA block. 
A

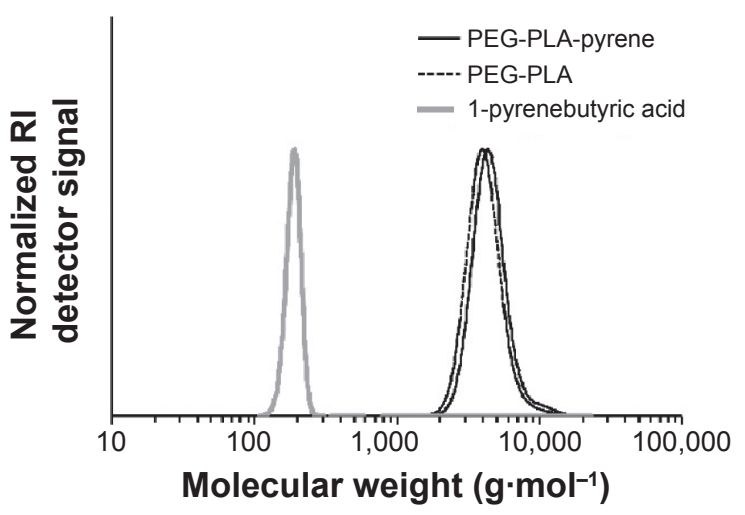

B

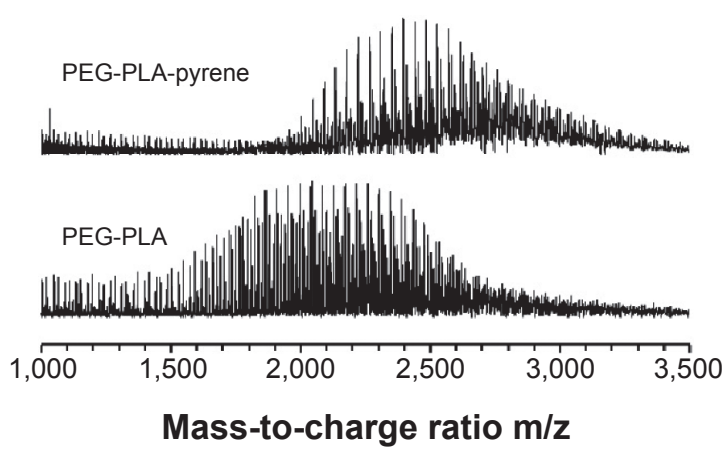

C ${ }^{1} \mathrm{H}-\mathrm{NMR}$

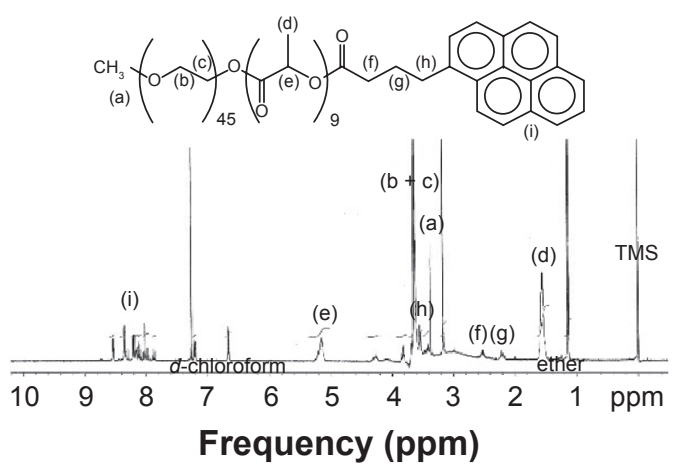

D

IR

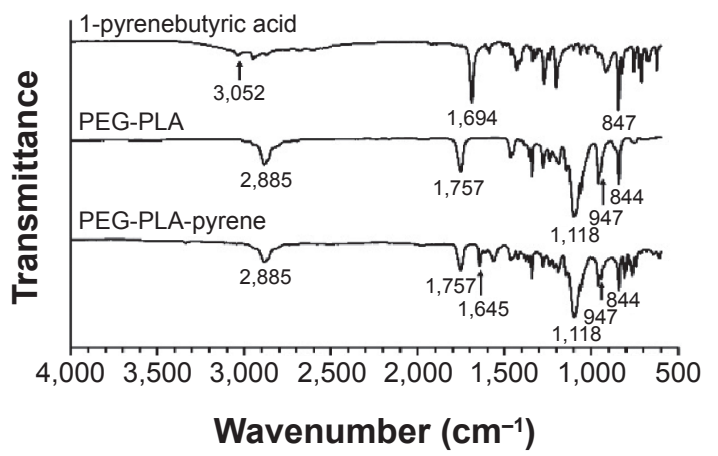

Figure 2 Characterization of PEG-PLA-pyrene by (A) GPC, (B) MALDI-TOF, (C) 'H-NMR, and (D) IR.

Abbreviations: GPC, gel permeation chromatography; 'H-NMR, proton nuclear magnetic resonance; IR, infrared spectroscopy; MALDI-TOF, matrix-assisted laser desorption/ ionization time-of-flight mass spectrometry; PEG-PLA, poly(ethylene glycol)-polylactide; RI, refractive index; TGA, thermogravimetric analysis; TMS, tetramethylsilane.

and the band at $847 \mathrm{~cm}^{-1}$ was due to aromatic stretching. ${ }^{12}$ On the PEG-PLA spectrum, the bands at 2,885 and $1,118 \mathrm{~cm}^{-1}$ were assigned to $\mathrm{C}-\mathrm{H}$ and $\mathrm{C}-\mathrm{O}-\mathrm{C}$ stretching, and those at 947 and $844 \mathrm{~cm}^{-1}$ were due to the crystalline phase of PEG. ${ }^{13}$ Another C-O stretching band appeared at $1,757 \mathrm{~cm}^{-1}$, in agreement with the presence of PLA blocks. ${ }^{13}$ On the PEG-PLA-pyrene spectrum, the stretching vibration of the conjugated $\mathrm{C}=\mathrm{O}$ group shifted to $1,645 \mathrm{~cm}^{-1}$, in agreement with the presence of carboxyl pyrene. Moreover, the $\mathrm{OH}$ stretching peak of 1-pyrenebutyric acid at 3,052 $\mathrm{cm}^{-1}$ disappeared in the PEG-PLA-pyrene spectrum, which proved the complete esterification reaction of fluorescence probe labeling.

Studies have been performed to prepare PLA-pyrene or PEG-PLA-pyrene conjugates. ${ }^{4,8}$ To synthesize PLA-pyrene conjugates, it is necessary to prepare the aldehyde-terminal PLA that can react with aminopyrene in the presence of sodium cyanoborohydride. ${ }^{8}$ To prepare the PEG-PLA-pyrene conjugate, pyrene-1-carbonyl cyanide was conjugated to the $\omega$-hydroxyl group of the PLA end of the PEG-PLA copolymer, using quinuclidine as the catalyst. ${ }^{4}$ However, the use of toxic materials could be a critical issue in the synthesis of biomaterials. Instead, we introduced a simpler labeling method using the carboxyl group of 1-pyrenebutyric acid coupled with the hydroxyl group of PEL-PLA in the presence of DIC/HOAt. The latter is the coupling agent commonly used in peptide synthesis.

The thermal properties of PEG-PLA-pyrene were investigated using DSC and TGA and were compared with the corresponding PEG-PLA and carboxyl pyrene. For DSC measurements, samples were first heated from room temperature to $105^{\circ} \mathrm{C}$ and then cooled to $30^{\circ} \mathrm{C}$, followed by a second heating to $220^{\circ} \mathrm{C}$ at a heating rate of $10^{\circ} \mathrm{C} \cdot \mathrm{min}^{-1}$. DSC thermograms of carboxyl pyrene, PEG-PLA, and PEG-PLApyrene are shown in Figure 3A. PEG-PLA-pyrene appeared semicrystalline with two melting peaks. The melting enthalpy $\left(\Delta \mathrm{H}_{\mathrm{m}}\right)$ of PEG-PLA-pyrene was found to be $100 \mathrm{~J} \cdot \mathrm{g}^{-1}$ in the first peak and $9 \mathrm{~J} \cdot \mathrm{g}^{-1}$ in the second peak. PEG-PLA showed a melting temperature $\left(\mathrm{T}_{\mathrm{m}}\right)$ value close to that of the first peak of PEG-PLA-pyrene. This finding showed that the presence 
A

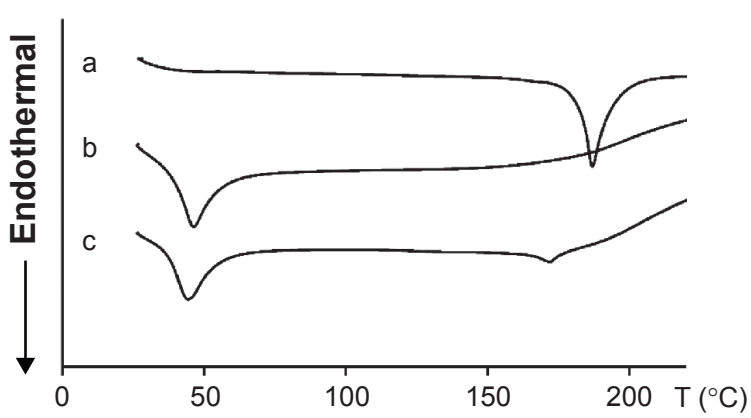

B

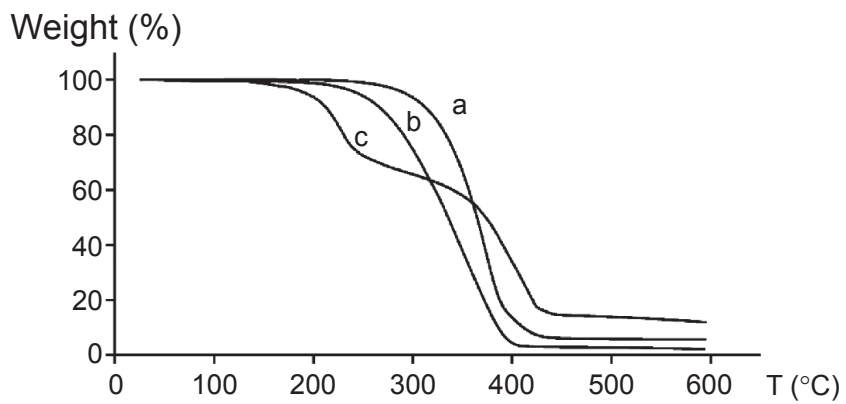

Figure 3 (A) DSC and (B) TGA thermograms of I-pyrenebutyric acid (a), PEG-PLA (b), and PEG-PLA-pyrene (c).

Abbreviations: DSC, differential scanning calorimetry; PEG-PLA, poly(ethylene glycol)-polylactide; TGA, thermogravimetric analysis.

of pyrene at the end of PLA did not influence the crystallization ability of the PEG blocks. However, the presence of PEG-PLA blocks strongly reduced the $\Delta \mathrm{H}_{\mathrm{m}}$ and $\mathrm{T}_{\mathrm{m}}$ of pyrene, indicating that the thermal characteristics were sensitive to PEG-PLA incorporation. TGA thermograms of the samples were registered from $30^{\circ} \mathrm{C}$ to $500^{\circ} \mathrm{C}$ at a heating rate of $10^{\circ} \mathrm{C} \cdot \mathrm{min}^{-1}$, as shown in Figure 3B. Pyrene and the PEGPLA copolymer exhibited a uniform thermal degradation behavior. In the case of the PEG-PLA-pyrene copolymer, a two-step degradation was detected. It is assumed that the first degradation step was due to the pyrene moiety. The value of decomposition temperature $\left(\mathrm{T}_{\mathrm{d}}\right)$ was read with respect to a $5 \%$ weight loss of the sample; thus, a $\mathrm{T}_{\mathrm{d}}$ value of $290^{\circ} \mathrm{C}$ was obtained for carboxyl pyrene, and $240^{\circ} \mathrm{C}$ and $190^{\circ} \mathrm{C}$ for the copolymers PEG-PLA and PEG-PLA-pyrene, respectively. The TGA curve of PEG-PLA-pyrene shifted slightly to a lower temperature compared with PEG-PLA and pyrene, ie, the overall stability decreased due to the coupling of PEGPLA segments and pyrene. From the processing viewpoint, the coupling of the PEG-PLA copolymer with pyrene provides a wide temperature range (from $50^{\circ} \mathrm{C}$ to $150^{\circ} \mathrm{C}$ ) for thermal treatments.

Characterization of the fluorescent properties of polymeric micelles was performed by dissolving $2.5 \mathrm{mg}$ polymer in $1 \mathrm{~mL}$ pure water followed by twofold serial dilutions of the sample. After sonication, we observed an abrupt enhancement in the ultraviolet absorption at 300 and $330 \mathrm{~nm}$ (Figure 4A), identifying an aromatic ring structure. In addition, the higher concentration of PEG-PLA-pyrene caused detection of increased absorbance intensity. Figure 3B shows the fluorescence emission spectra of serial diluted PEG-PLA-pyrene dispersions. Similar to the absorbance, as the PEG-PLA-pyrene component in the water solution increased, the intensity of the emission spectrum increased. However, no signal characteristics were detected on the absorbance and fluorescence spectra of PEG-PLA (Figure 4A and B). Due to the sensitivity of pyrene to the environmental polarity, the ratio of the two fluorescence intensities of the peak at 370 and $380 \mathrm{~nm}$ was plotted against the copolymer concentration. ${ }^{10}$ Figure $4 \mathrm{C}$ shows that the value of the fluorescence intensity ratio inverted at $1 \mathrm{mg} \cdot \mathrm{mL}^{-1}$, suggesting that the formation changes from an aqueous environment to the hydrophobic core of the micelles. ${ }^{1,4}$ The results are similar to the fluorescence spectra of PEG-PLA-pyrene in water, reported in the literature. ${ }^{4}$ Amphiphilic copolymers generally present a core-shell micellar architecture with a core consisting of hydrophobic blocks and a shell composed of chains. ${ }^{1}$ DLS measurements were performed to confirm the micelle sizes. The data showed that the PEGPLA polymeric aqueous solution possessed micelles with a unimodal distribution, with an average diameter of $9 \pm 3 \mathrm{~nm}$. It appears that coupling the pyrene moiety at the end of PLA did not significantly increase the size or size distribution of the polymeric micelles $(11 \pm 1 \mathrm{~nm})$. It has been shown that several pathways have been verified in the internalization of exogenous antigens by dendritic cells (DCs), such as phagocytosis, and receptor-mediated endocytosis and macropinocytosis. ${ }^{14}$ The latter is used to internalize extracellular fluid and smaller solutes, such as macromolecules and, particularly, small nanoparticles $(<50 \mathrm{~nm}) .{ }^{15}$ To investigate the internalization of PEG-PLA-pyrene micelles by DCs, PEG-PLA-pyrene was incubated with murine bone marrow-derived DCs (BMDCs) for 5, 10, 20, or 30 minutes (Figure S1). At $37^{\circ} \mathrm{C}$, the major internalized PEG-PLApyrene colocalized with early endosomes (a membrane-bound compartment inside of eukaryotic cells) after a 10-minute incubation with BMDCs. However, the internalized PEGPLA-pyrene was undetected in early endosomes until a 30-minute incubation with BMDCs at $4^{\circ} \mathrm{C}$; the internalization of PEG-PLA-pyrene by BMDCs is a temperature-dependent 
A
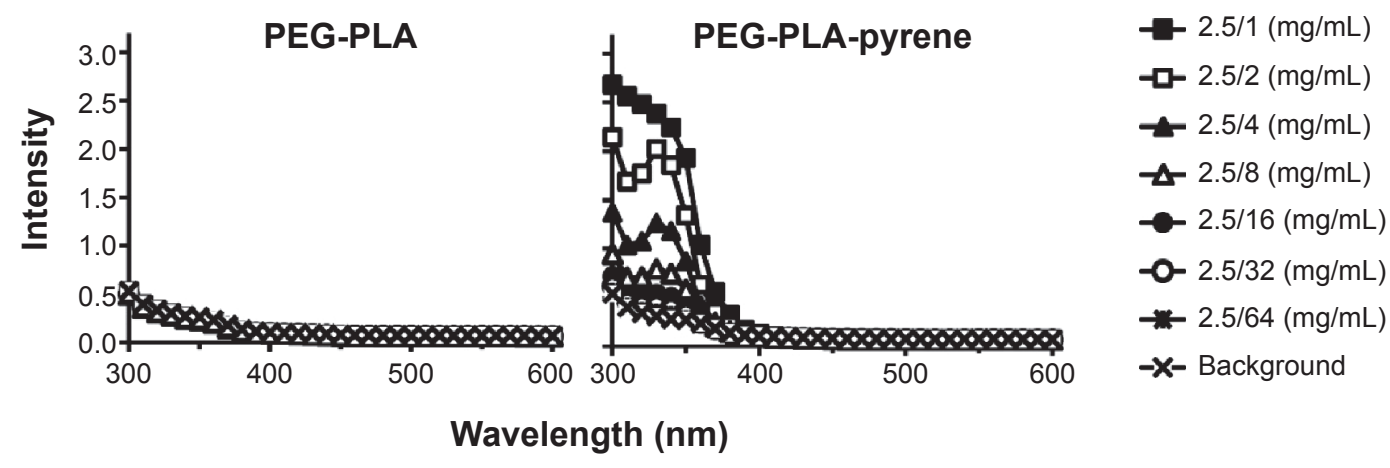

B
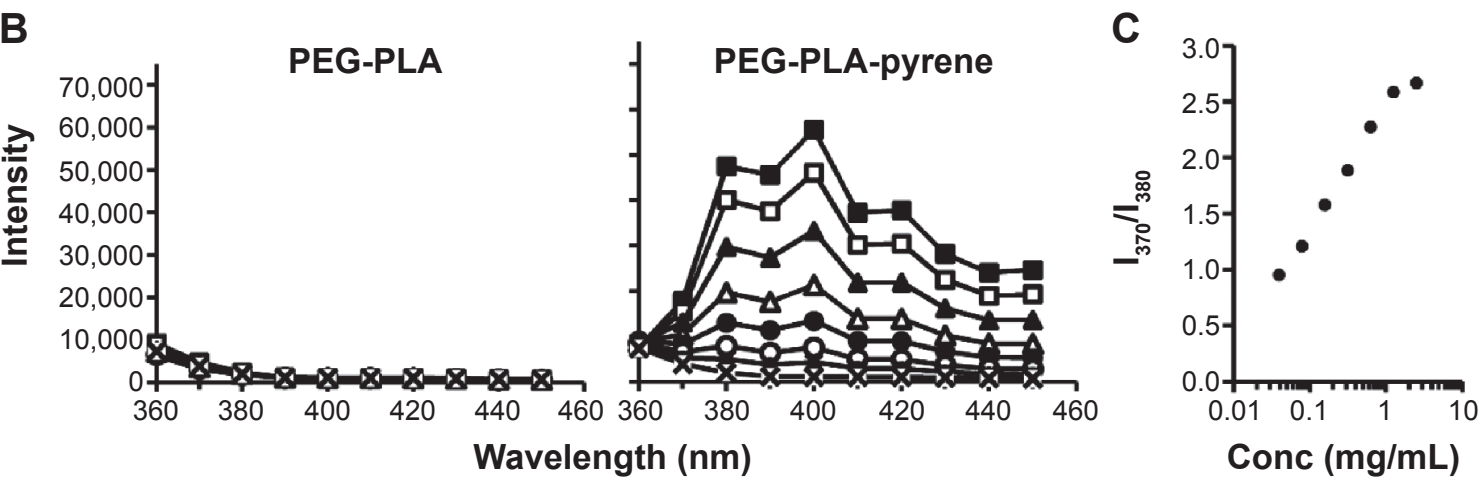

Figure 4 (A) UV-vis absorbance and (B) fluorescence emission spectra of PEG-PLA and PEG-PLA-pyrene in aqueous solutions of various concentrations, and (C) plot of the fluorescence intensity ratios $\left(\mathrm{I}_{370} / \mathrm{I}_{380}\right)$ as a function of concentration in aqueous media from PEG-PLA-pyrene.

Abbreviations: $I_{370} / I_{380}$, fluorescence intensity ratio of the peak at $370 \mathrm{~nm}$ to the peak at $380 \mathrm{~nm}$; PEG-PLA, poly(ethylene glycol)-polylactide; UV-vis, ultraviolet-visible.

process. Generally, the UV-vis absorbance and fluorescence emission intensities of PEG-PLA-pyrene in aqueous solution are concentration-dependent - this is a feature of great interest for the use of the conjugate as a fluorescence probe to study the in vivo distribution as well as the internalization and intracellular localization of polymeric micelles.

\section{Conclusion}

Pyrene-labeled biodegradable polymer PEG-PLA-pyrene was prepared by coupling carboxyl pyrene with a PEGPLA copolymer. Physicochemical characterization showed that the copolymer preserved the properties intrinsic to PEG-PLA; in addition, PEG-PLA-pyrene forms micelles in aqueous solutions. The results show that pyrene could serve as a useful fluorescence probe for attachment onto a polymer end group, for study of the in vivo distribution as well as the internalization and intracellular localization of polymeric micelles. These results also present a new approach to the synthesis of copolymers with functional end groups.

\section{Acknowledgments}

This study was supported by the 03A1-IVPP26-014 grant from the National Health Research Institutes of Taiwan and grant NSC-102-2320-B-400-001-MY2 from the Ministry of Science and Technology of Taiwan. The authors are grateful to Dr Yu-Cheng Chou, Institute of Biotechnology and Pharmaceutical Research of NHRI, for his help with the polymer characterization and to Dr Chung-Shi Yang, Institute of Biomedical Engineering and Nanomedicine of NHRI, for his help with the light scattering analysis.

\section{Disclosure}

The authors report no conflicts of interest in this work.

\section{References}

1. Jeong B, Bae YH, Lee DS, Kim SW. Biodegradable block copolymers as injectable drug-delivery systems. Nature. 1997;388(6645):860-862.

2. Zhang X, Li Y, Chen X, et al. Synthesis and characterization of the paclitaxel/MPEG-PLA block copolymer conjugate. Biomaterials. 2005; 26(14):2121-2128.

3. Yang L, Wu X, Liu F, Duan Y, Li S. Novel biodegradable polylactide/ poly(ethylene glycol) micelles prepared by direct dissolution method for controlled delivery of anticancer drugs. Pharm Res. 2009;26(10): $2332-2342$.

4. Jule E, Yamamoto Y, Thouvenin M, Nagasaki Y, Kataoka K. Thermal characterization of poly(ethylene glycol)-poly(D,L-lactide) block copolymer micelles based on pyrene excimer formation. J Control Release. 2004;97(3):407-419.

5. Zhang Y, Zhang Q, Zha L, et al. Preparation, characterization and application of pyrene-loaded methoxy poly(ethylene glycol)-poly(lactic acid) copolymer nanoparticles. Colloid Polym Sci. 2004;282:1323-1328. 
6. Siao SY, Lin LH, Chen WW, Huang MH, Chong P. Characterization and emulsifying properties of block copolymers prepared from lactic acid and poly(ethylene glycol). J Appl Polym Sci. 2009;114(1):509-516.

7. Chen WL, Liu SJ, Leng CH, Chen HW, Chong P, Huang MH. Disintegration and cancer immunotherapy efficacy of a squalane-in-water delivery system emulsified by bioresorbable poly(ethylene glycol)block-polylactide. Biomaterials. 2014;35(5):1686-1695.

8. Sasatsu M, Onishi H, Machida Y. Preparation and biodisposition of methoxypolyethylene glycol amine-poly(DL-lactic acid) copolymer nanoparticles loaded with pyrene-ended poly(DL-lactic acid). Int J Pharm. 2008;358(1-2):271-277.

9. Tan J, Li Y, Liu R, et al. Micellization and sustained drug release behavior of EC-g-PPEGMA amphiphilic copolymers. Carbohydr Polym. 2010;81(2):213-218.

10. Goddard ED, Turro NJ, Kuo PL, Ananthapadmanabhan KP. Fluorescence probes for critical micelle concentration determination. Langmuir. $1985 ; 1(3): 352-355$.
11. Bains G, Patel AB, Narayanaswami V. Pyrene: a probe to study protein conformation and conformational changes. Molecules. 2011;16(9): 7909-7935.

12. Majeed S, Filiz V, Shishatskiy S, Wind J, Abetz C, Abetz V. PyrenePOSS nanohybrid as a dispersant for carbon nanotubes in solvents of various polarities: its synthesis and application in the preparation of a composite membrane. Nanoscale Res Lett. 2012;7(1):296.

13. Huang MH, Li S, Coudane J, Vert M. Synthesis and characterization of block copolymers of $\varepsilon$-caprolactone and DL-lactide initiated by ethylene glycol or poly(ethylene glycol). Macromol Chem Phys. 2003; 204(16):1994-2001.

14. Reddy ST, Swartz MA, Hubbell JA. Targeting dendritic cells with biomaterials: developing the next generation of vaccines. Trends Immunol. 2006;27(12):573-579.

15. Song YC, Chou AH, Homhuan A, et al. Presentation of lipopeptide by dendritic cells induces anti-tumor responses via an endocytosisindependent pathway in vivo. J Leukoc Biol. 2011;90(2):323-332. 


\section{Supplementary material}

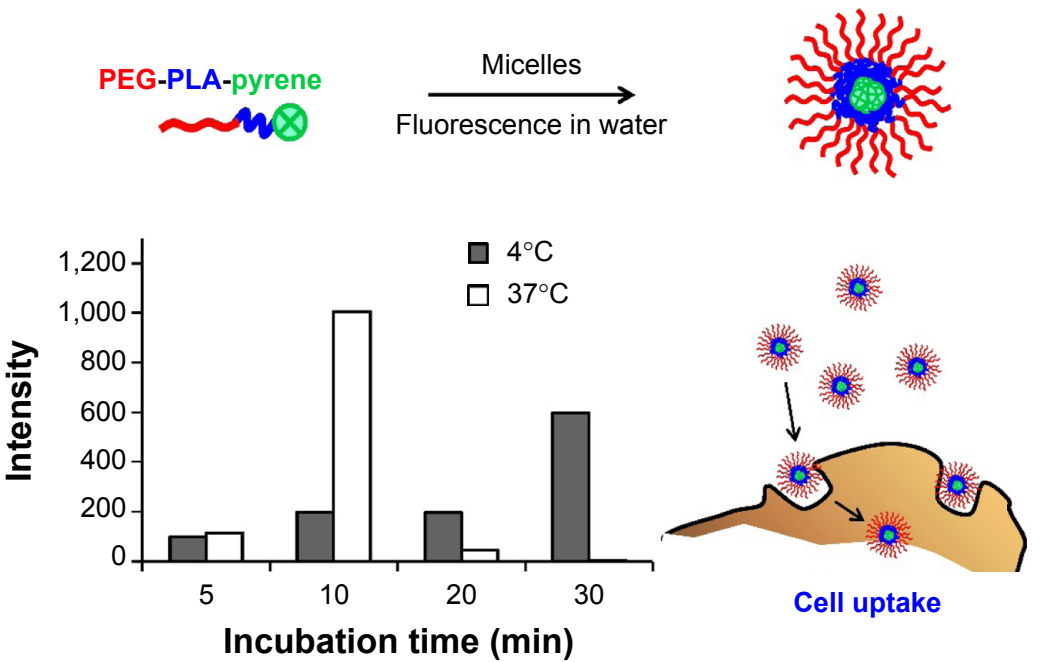

Figure SI Internalization of PEG-PLA-pyrene by BMDCs.

Notes: BMDCs from C57BL/6 mice were harvested and seeded at a density of $1 \times 10^{6}$ cells. Cells were then pulsed with $2 \mu \mathrm{g} \mathrm{mL}^{-1}$ of PEG-PLA-pyrene and incubated at $4^{\circ} \mathrm{C}$ and $37^{\circ} \mathrm{C}$. At different time points $(5,10,20$, and 30 minutes), cells were washed with cold medium, fixed with paraformaldehyde, and read by UV-visible absorption spectroscopy at $340 \mathrm{~nm}$. At $37^{\circ} \mathrm{C}$, the major internalized PEG-PLA-pyrene colocalized with early endosomes after a I0-minute incubation with BMDCs. However, internalized PEG-PLA-pyrene was undetected in early endosomes until a 30 -minute incubation with BMDCs at $4^{\circ} \mathrm{C}$; the internalization of PEG-PLA-pyrene by BMDCs is a temperaturedependent process. Generally, PEG-PLA-pyrene could serve as a useful fluorescence probe for studying the internalization and intracellular localization of polymeric micelles. The data are representative of two independent experiments.

Abbreviations: BMDC, bone marrow-derived dendritic cell; PEG-PLA, poly(ethylene glycol)-polylactide; UV, ultraviolet.

\section{Publish your work in this journal}

The International Journal of Nanomedicine is an international, peerreviewed journal focusing on the application of nanotechnology in diagnostics, therapeutics, and drug delivery systems throughout the biomedical field. This journal is indexed on PubMed Central, MedLine, CAS, SciSearch $®$, Current Contents ${ }^{\circledR} /$ Clinical Medicine,
Journal Citation Reports/Science Edition, EMBase, Scopus and the Elsevier Bibliographic databases. The manuscript management system is completely online and includes a very quick and fair peer-review system, which is all easy to use. Visit http://www.dovepress.com/ testimonials.php to read real quotes from published authors. 